INP MSU Preprint-95-2/366

Journal-ref: Int. J. of Mod. Phys. C7, No.6 (1996) pp.761-774

18 December 1996

\title{
RECURSIVE ALGORITHM FOR THE GENERATION OF RELATIVISTIC KINEMATICS FOR COLLISIONS AND DECAYS WITH REGULARIZATIONS OF SHARP PEAKS
}

\author{
V.A. Ilyin, D.N. Kovalenko, A.E. Pukhov \\ Institute of Nuclear Physics of Moscow State University, 119899 Moscow, Russia
}

\begin{abstract}
We describe an algorithm for the generation of relativistic kinematics for collision and decay processes with multiparticle final states. In the framework of this algorithm it is possible to generate different kinematics covering most of practically interesting cases. One gets a possibility to introduce different sets of integration variables. As a result different sets of kinematical singularities can be regularized. To smooth sharp peaks some regularization formulas and procedures are used covering most typical cases. The algorithm is realized in the package CompHEP created for automatic calculations of collision and decay processes.
\end{abstract}




\section{Introduction}

To evaluate numerically cross sections and other characteristics of elementary particle collisions and to calculate decays of unstable particles it is necessary to integrate squared matrix element over the phase space.

The first step is to express the integrand in terms of independent kinematical variables - integration variables. Note that after evaluation of Feynman diagrams the integrand is expressed in terms of scalar products of Lorentz momenta of in- and out-particles, and polarization vectors.

The next step is connected with a singular behavior of the integrand that is typical case in modern collider physics calculations (see Ref. [i], for example). In many cases straightforward methods of the numerical integration fail because of sharp peaks. Standard procedure to treat carefully such peaks is to transform integration variables in order to get nonsingular function in the new variables. We will call such procedure the regularization.

These two steps are closely connected. To make it clear let us note that singularities appear as zeros (more exactly - almost zeros) of propagators denominators. So one can say that each singularity is connected directly with a squared Lorentz 4-vector expressed in its order in terms of linear combination of particle momenta. However it is clear that singularity can be smoothed by integration variable transformation only if the corresponding invariant is one of these variables or related directly to such variable. Thus one can integrate carefully if both steps will be performed in proper relation to the set of singularities. This problem becomes nontrivial especially in so-called automatic calculation of collision and decay processes. This new approach, based on high perfomance and user-friendly programs, is developed now intensively Refs. [2]-[4]. With the help of these programs one can calculate different processes in the framework of the various physical models. It is clear that the generation of kinematics is an important part of this approach.

There are many known kinematical schemes implemented in existing approaches (see Ref. [5], for example). In GRACE [3] special library of kinematics, corresponded to different sets of kinematical singularities, was created. In EXCALIBUR [6] a multichannel appproach to compute four fermion processes in $e^{+} e^{-}$annihilation is presented. In this paper we demonstrate that the problem discussed can be treated in the framework of some general algorithm based on the recursive representation of kinematics as a tree of elementary decays $1 \rightarrow 2$. It opens the possibility to generate kinematics (choose integration variables, express squared matrix elements in terms of these variables and introduce proper regularizations) for almost all kinds of processes with the help of one program with a high level of user's interface. This algorithm was realized in the package CompHEP [2] and representative computational examples are given here.

\section{Kinematics}

By definition, the kinematical scheme is a way of phase space parameterization.

We define the kinematical scheme as a tree of elementary decays. Under the elementary decay we understand the parameterization in 2-particle phase space. Note that the words elementary decay do not correspond to real $1 \rightarrow 2$ decay in most cases. We use this terminology for convenience.

Below we consider multiparticle phase space for collisions. For decays the corresponding formulas can be written in a similar way.

Let us take any two out-particles with the numbers $i$ and $j$. We consider their momenta as momenta produced in the following elementary decay:

$$
q_{i j} \rightarrow q_{i}, q_{j}
$$

where $q_{i j}=q_{i}+q_{j}=\left(E_{i j}, \vec{q}_{i j}\right)$. Let us introduce a parameterization for this elementary decay in the rest frame of decayed 4 -vector $q_{i j}$ as

$$
d \Gamma_{2}(i, j) \equiv \frac{R_{i}}{(2 \pi)^{3} \cdot 4 \sqrt{s_{i j}}} \cdot d s_{i j} \cdot d \Omega_{i} .
$$

Here the measure $d \Omega_{i}$ corresponds to the angular part of $d \vec{q}_{i}$. Then $R_{i}=\left|\vec{q}_{i}\right|$ in the rest frame of $q_{i j}$, and $s_{i j}=q_{i j}^{2}$.

We repeat now this construction recursively: on each step two cluster 4-vectors $q_{I}, q_{J}$ are taken from the rest part of out-momenta or from the decayed 4 -vectors $q_{I J}$ appeared on previous steps. After $(N-1)$ steps we get the following formula for differential cross section $\left(|M|^{2}\right.$ is a squared matrix element - the normalization corresponds to the conventions [7):

$$
d \sigma=\frac{\pi}{2 R_{1} \sqrt{s}}|M|^{2} \cdot d \Gamma_{2}^{*}\left(I_{1}, J_{1}\right) \cdot \prod_{i=2}^{N-1} d \Gamma_{2}\left(I_{i}, J_{i}\right),
$$


where the 2-particle phase space measure for the 1 st elementary decay $p_{1}+p_{2} \rightarrow q_{I_{1}}$, $q_{J_{1}}$ does not include integration over $s \equiv\left(p_{1}+p_{2}\right)^{2}$ (here $p_{1}$ and $p_{2}$ are momenta of colliding particles):

$$
d \Gamma_{2}^{*}\left(I_{1}, J_{1}\right) \equiv \frac{R_{I_{1}}}{(2 \pi)^{3} \cdot 4 \sqrt{s}} \cdot d \Omega_{I_{1}} .
$$

Here $R_{I_{1}} \equiv\left\{\left|\vec{q}_{I_{1}}\right|\right.$, in $\left.C M S\right\}$, by CMS we denote the kinematical frame of center mass system.

The angular measure $d \Omega_{I}$ can be written in terms of two angular coordinates of the 3 -vector $\vec{q}_{I}$. Let us introduce two reference 4-vectors $\mathcal{P}_{I}=\left(\mathcal{P}_{I}^{0}, \overline{\mathcal{P}}_{I}\right)$ and $\mathcal{A}_{I}=\left(\mathcal{A}_{I}^{0}, \overline{\mathcal{A}}_{I}\right)$ for some elementary decay in the rest frame of the decayed vector $q_{I J}$. So, let the polar coordinate of $\vec{q}_{I}$, the angle $\Theta_{I}$, be counted from the 3 -vector $\overline{\mathcal{P}}_{I}$. Then let us take the azimuth coordinate of $\vec{q}_{I}$ as the angle $\Phi_{I}$ between two planes $\left(\overline{\mathcal{P}}_{I}, \overline{\mathcal{A}}_{I}\right)$ and $\left(\overline{\mathcal{P}}_{I}, \vec{q}_{I}\right)$. As a result the measure $d \Omega_{I}$ takes the form

$$
d \Omega_{I}=d \cos \Theta_{I} \cdot d \Phi_{I}
$$

Taking the reference vectors as different combination of the momenta of in- and out-particles we can connect different Lorentz invariants with introduced integration variables. Let us consider, for example, elementary decay $q_{I J} \rightarrow q_{I}, q_{J}$ and $\mathcal{P}_{I}=\sum_{n} p_{n}$, where $p_{n}$ are momenta of some in- and out-particles. The relation between the angle $\Theta_{I}$ and the invariant $\left(\sum p_{n}+q_{I}\right)^{2}$ can be written in the following form:

$$
\left(\sum p_{n}+q_{I}\right)^{2}=\left(\sum p_{n}\right)^{2}+\left(q_{I}\right)^{2}+2\left(\sum p_{n}^{0}\right) \cdot q_{I}^{0}-2\left|\sum \vec{p}_{n}\right| \cdot\left|\vec{q}_{I}\right| \cos \Theta_{I} .
$$

In the following we use this relation to introduce regularizations of sharp peaks $\$$.

Let us introduce numbering of the elementary decays by the following rules:

1. initial elementary decay $\left(p_{1}+p_{2} \rightarrow q_{I_{1}}, q_{J_{1}}\right)$ has a number $i=1$;

2. all elementary decays on branches, growing from the clusters $I_{i}$ and $J_{i}$ of the $i$-th elementary decay, have numbers larger than $i$;

3. any elementary decay on the branch growing from the cluster $I_{i}$ has a number smaller than any elementary decay on the branch growing from the second cluster $J_{i}$ of the $i$-th elementary decay.

We will use a terminology 'previous elementary decay' if it has smaller number than the current one. In CompHEP realization the integrations in (3) are ordered in the correspondence with this numbering of elementary decays.

\subsection{Kinematics summary}

- The kinematical scheme is a tree of "elementary decays".

- The number of elementary decays equals $\left(N_{\text {out }}-1\right)$, where $N_{\text {out }}$ is a number of out-particles.

- Each elementary decay is defined by

- three 4-vectors: decayed $q_{I J}$ and two cluster vectors, $q_{I}$ and $q_{J}$, with $q_{I J}=q_{I}+q_{J}$;

- reference vector $\mathcal{P}_{I}$ which can be chosen from some combinations of particle momenta. These combinations have to be already defined as cluster momenta or decayed vectors in previous elementary decays.

- The vectors $q_{I J}$ and $\mathcal{P}_{I}$ has to be linearly independent.

- Each elementary decay $q_{I J} \rightarrow q_{I}, q_{J}$ adds three new integration variables:

- $s_{I J}$ - squared decayed vector $q_{I J}$;

- $\Theta_{I}$ and $\Phi_{I}$ - polar and azimuth angles of $\vec{q}_{I}$ counted from the reference 3-vectors $\overline{\mathcal{P}}_{I}$ and $\overline{\mathcal{A}}_{I}$ in the rest frame of $q_{I J}$. For decays or collisions with fixed energies of in-particles the 1st elementary decay gives only two independent variables, $\Theta_{1}$ and $\Phi_{1}$. In that case the number of integration variables equals $3 \cdot\left(N_{\text {out }}-1\right)-1=3 \cdot N_{\text {out }}-4$.

\footnotetext{
${ }^{1}$ The relation between angle $\Phi_{I}$ and some Lorentz invariant is more complicated because it includes also cos $\Theta$. Of course, it is possible to use the angle $\Phi_{I}$ for the regularization purposes. However, we tested such regularizations and found them to be not effective and we do not consider this possibility here. For integration over $\Phi_{I}$ we take some fixed reference vector $\overline{\mathcal{A}}_{I}$. It could be unit vector orthogonal to the collision axes, for example.
} 


\section{Invariant Cuts}

In the framework of the algorithm described above it is possible to introduce effectively kinematical cuts over some combinations of Lorentz momenta of in- and out-particles.

In general we can introduce an invariant cut in two ways. The first is more effective and it can be realized if the corresponding invariant is connected directly with one of the integration variables of kinematical scheme (e.g., as Eq. (6)). In this case the corresponding cut can be implemented by the limitation of the integration volume.

In other cases entered cuts can be applied by multiplication of squared matrix element on the corresponding step function. This way can be not so effective, especially in the case of strong cut when the integrand equals zero in almost all phase space volume.

\section{Regularization}

In some cases the squared matrix element has singularities when the integrand has sharp peak(s) in phase space and the standard Monte Carlo integrator (even adaptive one) could fail. The typical examples are given below.

\subsection{General regularization formula}

Let the integrand $F(x)$ to be represented as $F(x)=f(x) \cdot\left(g_{1}(x)+\cdots+g_{n}(x)\right)$, where $f(x)$ is a smooth function and functions $g_{i}(x)$ have sharp peaks. One can transform the integration variable in the following way:

$$
d x=\frac{1}{\sum_{i=1}^{n} g_{i}(x(\tilde{y}))} d \tilde{y}, \quad \tilde{y}(x)=\sum_{i=1}^{n} G_{i}(x) \equiv G(x),
$$

where integral functions are $G_{i}(x)=\int_{a}^{x} g_{i}\left(x^{\prime}\right) d x^{\prime}$. The function $x(\tilde{y}) \equiv G^{-1}(\tilde{y})$ we will find from the relation $\sum_{i=1}^{n} G_{i}(x)-\tilde{y}=0$ numerically. Also let us transform the integration volume to the interval $[0,1]: d \tilde{y}=$ $(G(b)-G(a)) d y, 0 \leq y \leq 1$.

As a result the integral can be rewritten as

$$
\int_{a}^{b} F(x) d x=\int_{0}^{1} f(x(y)) \cdot\left(\sum_{i=1}^{n} g_{i}(x(y))\right) J_{\{g\}}(y) d y,
$$

where Jacobian equals $J_{\{g\}}(y)=\frac{1}{\sum_{i=1}^{n} g_{i}(x(y))}(G(b)-G(a))$.

We see that the singular integral is transformed now to smooth integration of the function $f(x)$. Of course integrand can have more complicated functional structure then we have considered. However, if we know the structure of singularities it is not so difficult to write some model function of the form $\left(g_{1}(x)+\cdots+g_{n}(x)\right)$ which will match each singularity. And as a result the transformation of the integration variable described above will regularize the integrand. Here we would like to stress again that the structure of singularities can be derived easily from the analysis of denominators of calculated Feynman diagrams.

Note that it is possible to smooth several peaks over one variable appearing from the propagators of different Feynman diagrams. In this case the model singular function $\sum_{i=1}^{n} g_{i}(x)$ is constructed with $g_{i}(x)$ corresponding to separate peaks.

Then we consider the constant $g_{n}(x)=1$ as one of model function. It is necessary to avoid neglect of contributions of nonsingular areas after regularization.

\subsection{Types of regularizations}

\subsection{1 $t$-channel pole}

Let us consider a typical example when in some Feynman diagram the in-particle with momentum $p_{1}$ radiates the virtual particle with mass $m$ and after that transforms to the out-particle with momentum $p_{3}$. The corresponding propagator of the virtual particle is

$$
\frac{1}{t-m^{2}}, \quad t \equiv\left(p_{3}-p_{1}\right)^{2}
$$




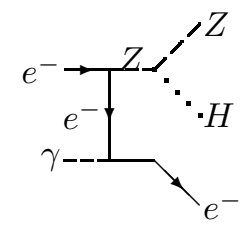

Figure 1: Feynman diagram of $\gamma+e^{-} \rightarrow e^{-}+Z+H$ with $t$-channel type of singularity, produced by the exchange of virtual electron.
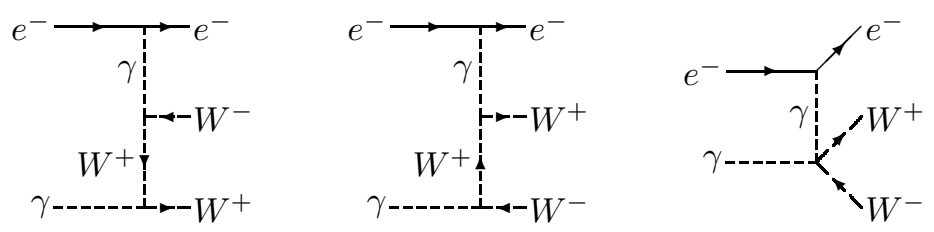

Figure 2: Feynman diagrams of $e^{-}+\gamma \rightarrow e^{-}+W^{+}+W^{-}$with $t$-channel type of singularity, produced by the exchange of virtual photon.

The integrand will have sharp peak in $t$ near its upper bound $t_{\max }$ if $m^{2}$ and $t_{\max }$ are much less than the energy of in-particles:

$$
m^{2} \ll s, \quad t_{\max } \ll s .
$$

Typical example is given in Fig. 11 by the Feynman diagram of the process

$$
\gamma\left(p_{1}\right)+e^{-}\left(p_{2}\right) \rightarrow e^{-}\left(p_{3}\right)+Z\left(p_{4}\right)+H\left(p_{5}\right),
$$

where $m=m_{e}$ is the electron mass, and we indicated momenta of particles in the brackets.

Another example is given by the process

$$
e^{-}\left(p_{1}\right)+\gamma\left(p_{2}\right) \rightarrow e^{-}\left(p_{3}\right)+W^{+}\left(p_{4}\right)+W^{-}\left(p_{5}\right),
$$

when $i n$-electron radiates the virtual photon (here $m=0$ ). The Feynman diagrams for this case are shown in Fig. 2.

Separate squared diagrams can include terms asymptotically proportional to $1 / t^{2}$ and also to $1 / t$. The $1 / t^{2}$ terms coming from different diagrams can cancel each other up to the terms of $1 / t$ type (in the $m_{e} \rightarrow 0$ limit) due to the gauge invariance. However, for processes $2 \rightarrow n$, with $n>2$, if the singular exchange propagator has its momentum composed from more than one out-particle momenta then there is no cancellation of $1 / t^{2}$ terms. For some processes of $2 \rightarrow 2$ type, like elastic scattering of two charged particles, there are no cancellation of $1 / t^{2}$ terms also. In these cases we have much stronger singularity.

Let us consider a $t$-channel singularity over $t_{I}=\left(p^{\text {in }}-\sum p_{i}^{\text {out }}\right)^{2}$, where $p^{\text {in }}$ is momentum of one of in-particles and sum is made over the some subset of out-particles. Suppose that we constructed a tree of elementary decays in such a way that we can choose some elementary decay, with clusters momenta $q_{1}$ and $q_{2}$, and the reference vector $\mathcal{P}$, as a combination of clusters momenta from previous elementary decays, according to one of two variants:

$$
\left(p^{\text {in }}-\sum p_{i}^{\text {out }}\right)^{2}=\left(q_{1}+\mathcal{P}\right)^{2}, \quad \text { or } \quad\left(p^{\text {in }}-\sum p_{i}^{\text {out }}\right)^{2}=\left(q_{2}+\mathcal{P}\right)^{2} .
$$

In these cases we have

$$
t_{I}=(q)^{2}+(\mathcal{P})^{2}+2\left(q_{0} \mathcal{P}_{0}-|\vec{q}||\overline{\mathcal{P}}| \cos \Theta\right), \quad q=q_{1,2}
$$

So, the invariant $t_{I}$ will be linear function of the integration variable $\cos \Theta$.

In the case of cancellation of $1 / t^{2}$ terms the model singular function is

$$
g_{i}(\cos \Theta)=\frac{1}{\cos \Theta-C_{0}} .
$$




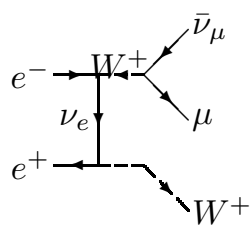

Figure 3: Feynman diagram with decayed $W$ propagator contributed in the process $e^{-}+e^{+} \rightarrow \bar{\nu}_{\mu}+\mu+W^{+}$.

Its integral function is

$$
G_{i}(\cos \Theta)=\ln \left|\cos \Theta-C_{0}\right|,
$$

where

$$
C_{0}=\frac{m^{2}-(\mathcal{P})^{2}-(q)^{2}+2 q_{0} \mathcal{P}_{0}}{2|\overline{\mathcal{P}}||\vec{q}|} .
$$

Here $m^{2}$ is the squared mass of the corresponding singular propagator (Eq. (90)).

In the cases without cancellation of $1 / t^{2}$ terms the model singular function is

$$
g_{i}(\cos \Theta)=\frac{1}{\left(\cos \Theta-C_{0}\right)^{2}},
$$

with integral function

$$
G_{i}(\cos \Theta)=\frac{1}{C_{0}-\cos \Theta} .
$$

\subsection{2 $s$-channel decay pole}

When a process includes the $s$-channel decay of some unstable particle it is necessary to introduce a finite width in the corresponding propagator to get a physically meaningful (and finite) cross section. Such diagrams will give in squared matrix element the factor

$$
\frac{1}{\left(s_{k}-M^{2}\right)^{2}+\Gamma^{2} \cdot M^{2}},
$$

where $M$ is the mass of unstable particle, $\Gamma$ is its width and $s_{k}$ is the squared invariant mass of decay products. The integrand has a sharp peak if

$$
\Gamma \cdot M \ll s .
$$

An example is the process

$$
e^{-}+e^{+} \rightarrow \bar{\nu}_{\mu}+\mu+W^{+}
$$

which includes the $W^{-}$decay into the lepton pair $\bar{\nu}_{\mu} \mu$. The Feynman diagram with such singularity is shown in Fig. 3 .

If $s_{k}$ is an integration variable (so it is squared decayed momentum of one elementary decay of the kinematical scheme) the corresponding model singular function can be taken as

$$
g_{i}\left(s_{k}\right)=\frac{1}{\left(s_{k}-M^{2}\right)^{2}+\varepsilon^{2}}
$$

and its integral function equals

$$
G_{i}\left(s_{k}\right)=\frac{1}{\varepsilon} \arctan \left(\frac{s_{k}-M^{2}}{\varepsilon}\right),
$$

where $\varepsilon \equiv \Gamma \cdot M$.

Let us now consider the case when in some kinematical scheme $s_{k}$ does not correspond to decayed momentum of any elementary decay. So we can not regularize this singularity by the previous way. To carry out the regularization in this case we need to choose elementary decay, with that 4-momenta $q_{1}$ and $q_{2}$ of its two 

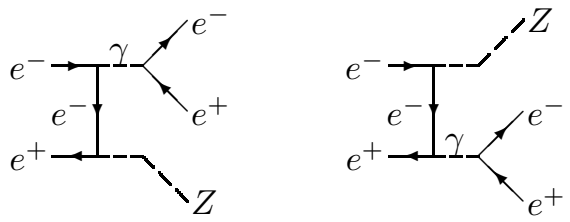

Figure 4: Feynman diagrams for $e^{-}+e^{+} \rightarrow e^{-}+e^{+}+Z$ with infrared type of kinematical singularity.

clusters, and the reference vector $\mathcal{P}$, as a combination of clusters momenta from previous elementary decays, according to one of the following variants:

$$
s_{k}=\left(\sum p_{i}^{\text {out }}\right)^{2}=\left(q_{1}+\mathcal{P}\right)^{2}, \quad \text { or } \quad s_{k}=\left(\sum p_{i}^{\text {out }}\right)^{2}=\left(q_{2}+\mathcal{P}\right)^{2} .
$$

In these cases we have

$$
s_{k}=(q)^{2}+(\mathcal{P})^{2}+2\left(q_{0} \mathcal{P}_{0}-|\vec{q}||\overline{\mathcal{P}}| \cos \Theta\right), \quad q=q_{1,2} .
$$

Thus the invariant $s_{k}$ will be linear function of the integration variable $\cos \Theta$. The model singular function for such cases is

$$
g_{i}(\cos \Theta)=\frac{1}{\left(\cos \Theta-C_{0}\right)^{2}+\varepsilon_{0}^{2}}
$$

and its integral function equals

$$
G_{i}(\cos \Theta)=\frac{1}{\varepsilon_{0}} \arctan \left(\frac{\cos \Theta-C_{0}}{\varepsilon_{0}}\right)
$$

where

$$
C_{0}=\frac{M^{2}-(q)^{2}-(\mathcal{P})^{2}-2 q_{0} \mathcal{P}_{0}}{-2|\vec{q}||\overline{\mathcal{P}}|}
$$

and

$$
\varepsilon_{0}=\frac{\varepsilon}{-2|\vec{q}||\overline{\mathcal{P}}|}
$$

\subsubsection{Infrared type of the singularity}

Let two light out-particles with masses $m_{1}$ and $m_{2}$ (for instance photon, electron, u or $\mathrm{d}$ quarks) be "products of the decay" of some virtual particle with mass $m$, and $m<m_{1}+m_{2}$. Sharp peak arises from the propagator of the "decaying" virtual particle with invariant mass $s_{i}$ :

$$
\frac{1}{\left(s_{i}-m^{2}\right)^{2}}, \quad s_{i} \geq\left(m_{1}+m_{2}\right)^{2},
$$

if the masses $m_{1}, m_{2}$, and $m$ are small:

$$
m_{1}, m_{2}, m \ll \sqrt{s} .
$$

Note that in the Standard Model such singular structure appears typically if one of these three particles is photon or gluon. An example is a "decay" of virtual photon into two light charged particles. Such peak arises in some Feynman diagrams (see Fig. (1) of the process

$$
e^{-}+e^{+} \rightarrow e^{-}+e^{+}+\mathbf{Z} .
$$

In this case $m=0$ and $m_{1}=m_{2}=m_{e}$ - mass of electron.

The singularity of infrared type is present also in the case of out-photon emission from the charged outparticle. In this case $m_{1}=0$ and $m_{2}=m$ is a mass of charged particle. The total cross section is infinite in this case - there is a nonintegrable pole at zero photon energy (so-called infrared catastrophe). However one can introduce the cut

$$
s_{i}>\delta^{2}+m^{2}, \quad s_{i}=\left(p_{m_{1}}+p_{m_{2}}\right)^{2},
$$


where $p_{m_{1}}$ and $p_{m_{2}}$ are momenta of the out-photon and charged out-particle. In this case sharp peak appears if

$$
\delta \ll \sqrt{s} .
$$

All these situations are different from the previous example of $s$-channel singularity. First, the pole is placed beyond the physical region. Second, due to the gauge invariance, the singularity will be of the first order, terms of $1 /\left(s_{i}-m^{2}\right)^{2}$ type will always cancel each other up to the terms of $1 /\left(s_{i}-m^{2}\right)$ type. So we have the similar functional problem as in the case of $t$-channel pole and we can use the formulas of the $t$-channel regularization to handle such poles.

\section{Computational Examples}

The method described in the previous sections was realized in CompHEP [2] package. Let us inspect the efficiency of this method in some examples. The results of cross section calculations by CompHEP with Monte Carlo integration are listed below. The program VEGAS [8] was used for multidimensional adaptive MC integration.

\section{$5.1 \quad t$-channel singularity}

Let us begin from the process

$$
e^{-}\left(p_{1}\right)+\gamma\left(p_{2}\right) \rightarrow e^{-}\left(p_{3}\right)+Z\left(p_{4}\right)+H\left(p_{5}\right)
$$

with singularity appearing from the exchange of electron in $t$-channel (Fig. 1). First let us use the default kinematics in CompHEP:

$$
\begin{array}{ll}
\text { 1st elementary decay: } & p_{1}+p_{2} \rightarrow p_{3}, p_{4}+p_{5} \text { with } \mathcal{P}=p_{1} ; \\
\text { 2nd elementary decay: } & p_{4}+p_{5} \rightarrow p_{4}, p_{5} \text { with } \mathcal{P}=-p_{1}+p_{3} .
\end{array}
$$

\begin{tabular}{|c|c|c|c|c|c|}
\hline \multirow[t]{2}{*}{ IT } & \multicolumn{2}{|l|}{ CURRENT RESULTS } & \multicolumn{2}{|c|}{ ACCUMULATED RESULTS } & \multirow[t]{2}{*}{$\mathrm{CHI} * * 2$} \\
\hline & Cross section [pb] & Error \% & Cross section [pb] & Error \% & \\
\hline 1 & $3.685360 \mathrm{E}-04$ & 24.68 & $3.685360 \mathrm{E}-04$ & 24.68 & .00 \\
\hline 2 & $6.255344 \mathrm{E}-04$ & 27.15 & $4.258167 \mathrm{E}-04$ & 18.83 & 1.78 \\
\hline 3 & $4.638301 \mathrm{E}-04$ & 10.09 & $4.541736 \mathrm{E}-04$ & 8.90 & .97 \\
\hline 4 & $5.303054 \mathrm{E}-04$ & 6.19 & $5.000283 E-04$ & 5.10 & 1.36 \\
\hline 5 & $6.214947 \mathrm{E}-04$ & 16.34 & $5.072243 E-04$ & 4.87 & 1.36 \\
\hline 6 & $6.355495 \mathrm{E}-04$ & 8.07 & $5.314256 \mathrm{E}-04$ & 4.19 & 2.10 \\
\hline 7 & $7.458411 \mathrm{E}-04$ & 7.14 & $5.633162 \mathrm{E}-04$ & 3.65 & 4.05 \\
\hline 8 & $9.273354 \mathrm{E}-04$ & 19.66 & $5.678777 \mathrm{E}-04$ & 3.59 & 4.04 \\
\hline 9 & $8.410235 \mathrm{E}-04$ & 6.03 & $6.059285 \mathrm{E}-04$ & 3.13 & 6.65 \\
\hline 10 & $7.566958 \mathrm{E}-04$ & 1.20 & $7.285007 \mathrm{E}-04$ & 1.12 & 11.64 \\
\hline
\end{tabular}

For the number of points per iteration equals 10000, we can see the following CompHEP screen output for the total cross section calculation at $\sqrt{s}=500 \mathrm{GeV}$ :

Here IT is iteration number, CURRENT RESULTS are results of the integration for current iteration. ACCUMULATED RESULTS are cumulative value of the integration of current and previous iterations. These numbers, as well as the error and parameter $\chi^{2}$, are evaluated according standard formulas (see details in Ref. [8]).

The result above shows that there is no stability from iteration to iteration (see 2nd column - values of current results; and 3rd column — current errors). Also there is no reliability with due to large value of $\chi^{2}$ parameter.

Let us now introduce the kinematical regularization of the $t$-channel type for the invariant $\left(p_{3}-p_{2}\right)^{2}$ with mass $m=m_{e}$. Used kinematical scheme allows to introduce this regularization because $\mathcal{P}$ in the 1st elementary decay equals $p_{1}$ and $p_{1}=-p_{2}$ in the 1 st decay. The CompHEP screen output of the integration with entered regularization will be:

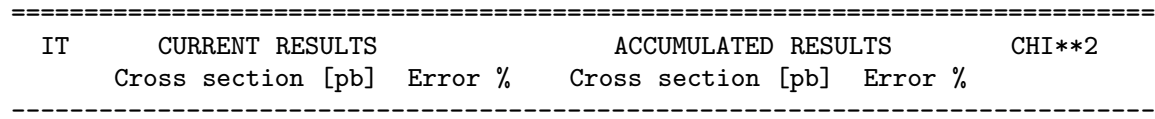




\begin{tabular}{rrrrrr}
1 & $7.621777 \mathrm{E}-04$ & .33 & $7.621777 \mathrm{E}-04$ & .33 & .00 \\
2 & $7.598378 \mathrm{E}-04$ & .21 & $7.605149 \mathrm{E}-04$ & .18 & .62 \\
3 & $7.590317 \mathrm{E}-04$ & .18 & $7.597725 \mathrm{E}-04$ & .13 & .61 \\
\hline
\end{tabular}

\section{$5.2 s$-channel singularity}

Let us consider an example with $s$-channel singularity (see Fig. 3):

$$
e^{-}\left(p_{1}\right)+e^{+}\left(p_{2}\right) \rightarrow \bar{\nu}_{\mu}\left(p_{3}\right)+\mu\left(p_{4}\right)+W^{+}\left(p_{5}\right) .
$$

If we use the following kinematical scheme

$$
\begin{array}{ll}
\text { 1st elementary decay: } & p_{1}+p_{2} \rightarrow p_{3}, p_{4}+p_{5} \text { with } \mathcal{P}=p_{2} \\
\text { 2nd elementary decay: } & p_{4}+p_{5} \rightarrow p_{4}, p_{5} \text { with } \mathcal{P}=p_{3},
\end{array}
$$

\begin{tabular}{|c|c|c|c|c|c|}
\hline \multirow[t]{2}{*}{ IT } & \multicolumn{2}{|l|}{ CURRENT RESULTS } & \multicolumn{2}{|c|}{ ACCUMULATED RESULTS } & \multirow[t]{2}{*}{$\mathrm{CHI} * * 2$} \\
\hline & Cross section [pb] & Error \% & Cross section [pb] & Error \% & \\
\hline 1 & $6.407551 \mathrm{E}-01$ & 27.60 & $6.407551 \mathrm{E}-01$ & 27.60 & .00 \\
\hline 2 & $8.139560 \mathrm{E}-01$ & 10.75 & $7.798860 \mathrm{E}-01$ & 10.06 & .77 \\
\hline 3 & $7.667456 \mathrm{E}-01$ & 3.48 & $7.681075 \mathrm{E}-01$ & 3.29 & .40 \\
\hline 4 & $8.065722 \mathrm{E}-01$ & 2.70 & $7.901984 \mathrm{E}-01$ & 2.08 & .71 \\
\hline 5 & $8.483987 \mathrm{E}-01$ & 4.06 & $8.010476 \mathrm{E}-01$ & 1.86 & 1.11 \\
\hline 6 & $8.442694 \mathrm{E}-01$ & 2.95 & $8.123698 \mathrm{E}-01$ & 1.57 & 1.33 \\
\hline 7 & $8.276117 \mathrm{E}-01$ & 2.50 & $8.165697 \mathrm{E}-01$ & 1.33 & 1.18 \\
\hline 8 & $8.402845 \mathrm{E}-01$ & 2.54 & $8.214562 \mathrm{E}-01$ & 1.18 & 1.15 \\
\hline 9 & $8.512594 \mathrm{E}-01$ & 3.52 & $8.242752 \mathrm{E}-01$ & 1.12 & 1.12 \\
\hline 10 & $8.232579 \mathrm{E}-01$ & 3.04 & $8.241540 \mathrm{E}-01$ & 1.05 & .99 \\
\hline
\end{tabular}

then the CompHEP screen output with 25000 points per iteration and $\sqrt{s}=500 \mathrm{GeV}$ is:

We see that the error of current iteration (see 3 rd column) is standing at the level about $3 \%$ after 10 iterations that it is not satisfactory in many applications.

Let us choose other kinematical scheme

\begin{tabular}{|c|c|c|c|c|c|}
\hline \multirow[t]{2}{*}{ IT } & \multicolumn{2}{|l|}{ CURRENT RESULTS } & \multicolumn{2}{|c|}{ ACCUMULATED RESULTS } & \multirow[t]{2}{*}{$\mathrm{CHI} * * 2$} \\
\hline & Cross section [pb] & Error \% & Cross section [pb] & Error \% & \\
\hline 1 & $9.342104 \mathrm{E}-01$ & 29.08 & $9.342104 \mathrm{E}-01$ & 29.08 & .00 \\
\hline 2 & $8.762828 \mathrm{E}-01$ & 8.78 & $8.805859 \mathrm{E}-01$ & 8.41 & .04 \\
\hline 3 & $8.677625 \mathrm{E}-01$ & 1.18 & $8.680033 \mathrm{E}-01$ & 1.17 & .04 \\
\hline 4 & $8.463662 \mathrm{E}-01$ & .65 & $8.513031 \mathrm{E}-01$ & .57 & 1.19 \\
\hline 5 & $8.471336 \mathrm{E}-01$ & .57 & $8.492184 \mathrm{E}-01$ & .40 & .99 \\
\hline
\end{tabular}

$$
\begin{array}{ll}
\text { 1st elementary decay: } & p_{1}+p_{2} \rightarrow p_{3}+p_{4}, p_{5} \text { with } \mathcal{P}=p_{2} \\
\text { 2nd elementary decay: } & p_{3}+p_{4} \rightarrow p_{3}, p_{4} \text { with } \mathcal{P}=p_{1} .
\end{array}
$$

One can see much better results even without regularization:

\begin{tabular}{|c|c|c|c|c|c|}
\hline \multirow[t]{2}{*}{ IT } & \multicolumn{2}{|l|}{ CURRENT RESULTS } & \multicolumn{2}{|c|}{ ACCUMULATED RESULTS } & \multirow[t]{2}{*}{$\mathrm{CHI} * * 2$} \\
\hline & Cross section [pb] & Error \% & Cross section [pb] & Error \% & \\
\hline 1 & $8.535685 \mathrm{E}-01$ & 4.72 & $8.535685 \mathrm{E}-01$ & 4.72 & .00 \\
\hline 2 & $8.539195 \mathrm{E}-01$ & .94 & $8.539060 \mathrm{E}-01$ & .93 & .00 \\
\hline 3 & $8.544500 \mathrm{E}-01$ & .60 & $8.542897 \mathrm{E}-01$ & .50 & .00 \\
\hline 4 & $8.449297 \mathrm{E}-01$ & .58 & $8.502279 \mathrm{E}-01$ & .38 & .69 \\
\hline 5 & $8.481124 \mathrm{E}-01$ & .57 & $8.495686 \mathrm{E}-01$ & .32 & .55 \\
\hline
\end{tabular}

Then introducing regularization over invariant mass $\left(p_{3}+p_{4}\right)^{2}$ with the mass parameter $M=M_{W}$ ( $W$-boson mass) and the width parameter $\Gamma=\Gamma_{W}$ ( $W$-boson width) we get more effective integration than in the previous case: 
The result of regularization is strong decrease of the error of first iterations.

However, it can happen that for some reason one cannot choose kinematical scheme where this $s$-channel singularity is related to some integration variable as an invariant mass of elementary decay. In the process $e^{-}+e^{+} \rightarrow e^{-}+\bar{\nu}_{e}+W^{+}$, for example, when discussed $s$-channel singularity contributes, other singularities are present and they can force us to introduce another kinematics to regularize these peaks. In such cases one can use regularization of the $s$-channel singularity using polar angle $\Theta$ (Eq. (22)). In our demonstration calculation let us return to the first kinematical scheme given at the beginning of this section and introduce the regularization for the corresponding $\left(p_{3}+p_{4}\right)^{2}$ invariant. It is possible to do so because $p_{3}$ has been choosen in this kinematical scheme as reference vector $\mathcal{P}$ in the 2 nd elementary decay. The CompHEP screen output for this case will be:

\begin{tabular}{|c|c|c|c|c|c|}
\hline \multirow[t]{2}{*}{ IT } & CURRENT RESULTS & \multicolumn{3}{|c|}{ ACCUMULATED RESULTS } & \multirow[t]{2}{*}{$\mathrm{CHI} * * 2$} \\
\hline & Cross section [pb] & Error \% & Cross section [pb] & Error \% & \\
\hline 1 & $8.854719 \mathrm{E}-01$ & 4.85 & $8.854719 \mathrm{E}-01$ & 4.85 & .00 \\
\hline 2 & $8.453821 \mathrm{E}-01$ & 1.00 & $8.468828 \mathrm{E}-01$ & .98 & .84 \\
\hline 3 & $8.477949 \mathrm{E}-01$ & .77 & $8.474495 \mathrm{E}-01$ & .60 & .42 \\
\hline 4 & 8.497170E-01 & .82 & $8.482457 \mathrm{E}-01$ & .49 & .30 \\
\hline 5 & $8.503980 \mathrm{E}-01$ & .75 & $8.488813 \mathrm{E}-01$ & .41 & .25 \\
\hline
\end{tabular}

One has to compare this screen output with the first one at the beginning of this section. We see that the integration is stable with fast convergency, smaller error and reliable value of $\chi^{2}$ parameter, similar to the previous case when the regularization over $\left(p_{3}+p_{4}\right)^{2}$ as invariant mass of elementary decay was performed.

\section{Acknowledgments}

This work was partially supported by European Association INTAS (grant 93-1180-ext), Russian Foundation for Basic Reseach (RFBR grant 96-02-19773a) and JSPS (Japan Society for Promotion Science).

We are grateful to E. E. Boos, M. N. Dubinin, and S. A. Shichanin for fruitful discussions and testing the program, and to Y. Shimizu for useful discussions.

\section{References}

[1] D. Bardin et al., "WW physics and generators," in Physics at LEP II, ed. G. Altarelli, T. Sjostrand, and F. Zwirner, CERN report 96-01, 1996.

[2] E. Boos et al., SNUTP 94-116 Seoul 1994, INP MSU-94-36/358 Moscow 1994; hep-ph/9503280.

[3] GRACE manual, KEK Report 92-19, 1993.

[4] J. Küblbeck, H. Eck, and R. Mertig, "Computeralgebraic generation and calculation of Feynman graphs using FeynArts and FeynCalc," in Proc. of the Second International Workshop on Software Engineering, Artificial Intelligence and Expert Systems in High Energy and Nuclear Physics, ed. D. Perret-Gallix (1992).

[5] E. Byckling and K. Kajantie, Particle Kinematics (John Wiley \& Sons, London, 1973).

[6] R. Kleiss and R. Pittau, "Weight optimization in multichannel Monte Carlo," NIKHEF-H 94-17 and INLO-PUB-4/94; F.A. Berends, R. Kleiss, and R. Pittau, "EXCALIBUR - a Monte Carlo program to evaluate all four fermion processes at LEP 200 and beyond," INLO-PUB-12/94.

[7] "Particle Data Group," Phys. Rev. D50, No. 3 (1994).

[8] G.P. Lepage, J. Comp. Phys. 27, 192 (1978). 